\title{
Novel characteristics of Chinese NADC34-like PRRRSV during 2020-2021
}

\author{
$\mathrm{Hu} \mathrm{Xu}^{1}$, Chao $\mathrm{Li}^{1}$, Wansheng $\mathrm{Li}^{1}$, Jing Zhao ${ }^{1}$, Bangjun Gong ${ }^{1}$, Qi Sun ${ }^{1}$, Yandong Tang ${ }^{1}$, \\ Lirun Xiang ${ }^{1}$, Chaoliang Leng ${ }^{2}$, Jinmei Peng${ }^{1}$, Qian Wang ${ }^{1}$, Fandan Meng ${ }^{1}$, Ying $\mathrm{Yu}^{3}$, \\ Tongqing $\mathrm{An}^{1}$, Xuehui Cai ${ }^{1}$, Zhi-Jun Tian ${ }^{1}$, and HongLiang Zhang ${ }^{1}$ \\ ${ }^{1}$ Chinese Academy of Agricultural Sciences Harbin Veterinary Research Institute \\ ${ }^{2}$ Nanyang Normal University Rothamsted Research Joint Laboratory of Insect Biology \\ ${ }^{3}$ Qingdao Agricultural University
}

December 26, 2021

\begin{abstract}
NADC34-like PRRSV strains were first detected in China in 2017, with epidemic potential. In this study, the phylogenetic, epidemic, and recombinant properties of NADC34-like PRRSV in China were evaluated comprehensively. From 2020 to October 2021, 82 NADC34-like PRRSV isolates were obtained from 433 PRRSV-positive clinical samples. These strains accounted for $11.5 \%$ and $28.6 \%$ of positives in 2020 and 2021, respectively, and have spread to eight provinces. We selected 15 samples for whole-genome sequencing, revealing genome lengths of 15,009 to 15,113 nt. Phylogenetic analysis revealed that Chinese NADC34-like strains cluster with American sublineage 1.5 strains and do not form an independent branch. Recombination analysis revealed that six of fifteen complete genome sequences derived from recombination between NADC34-like and NADC30like or HP-PRRSV; they all recombined with local strains in China, exhibiting a complex recombination pattern. Partial Nsp2 sequence alignment showed that nine of fifteen isolates have a continuous 100-aa deletion (similar to IA/2014/NADC34); other isolates have a 131-aa discontinuity deletion (similar to NADC30). Five of them also have additional amino acid deletions, all of which are reported for the first time here. In the last two years, NADC34-like PRRSV has become one of the main epidemic strains in some areas of China; it has changed significantly, its homology has decreased significantly, and it has undergone complex recombination with local Chinese strains. These results are of great significance for understanding the current epidemic situation of PRRSV in China.
\end{abstract}

Novel characteristics of Chinese NADC34-like PRRRSV during 2020-2021

$\mathrm{Hu} \mathrm{Xu}{ }^{\text {a1 }}$, Chao Li a 1, Wansheng $\mathrm{Li}^{\mathrm{a}}{ }^{1}$, Jing Zhao a, Bangjun Gonga ${ }^{\mathrm{a}}$, Qi Sun ${ }^{\mathrm{a}}$, Yan-dong Tang ${ }^{\mathrm{a}}$, Lirun Xiang $^{\mathrm{a}}$, ChaoLiang Leng ${ }^{\mathrm{b}}$, Jinmei Peng ${ }^{\mathrm{a}}$, Qian Wang ${ }^{\mathrm{a}}$, Fandan Meng ${ }^{\mathrm{a}}$, Ying $\mathrm{Yu}^{\mathrm{c}}$, Tongqing An ${ }^{\mathrm{a}}$, XueHui $\mathrm{Cai}^{\mathrm{a}}$, Zhi-Jun Tian ${ }^{\mathrm{a}}$, HongLiang Zhang ${ }^{\mathrm{a}^{*}}$

${ }^{a}$ State Key Laboratory of Veterinary Biotechnology, Harbin Veterinary Research Institute, Chinese Academy of Agricultural Sciences, Harbin 150001, China

${ }^{\mathrm{b}}$ Henan Key Laboratory of Insect Biology in Funiu Mountain, Henan Provincial Engineering Laboratory of Insects Bio-reactor, China-UK-NYNU-RRes Joint Laboratory of Insect Biology, Nanyang Normal University, Nanyang 473061, China

c Department of Preventive Veterinary Medicine, College of Veterinary Medicine, Qingdao Agricultural University, Qingdao, 266109, China

1 These authors contributed equally to this work. 
* Corresponding author contact: Harbin Veterinary Research Institute, CAAS, No. 678 Haping Road, Xiangfang District, Harbin 150001, China.

E-mail address: tianzhijun@caas.com

** Corresponding author contact: Harbin Veterinary Research Institute, CAAS, No. 678 Haping Road, Xiangfang District, Harbin 150001, China.

Tel.: +86-13624503578

E-mail address:zhanghongliang01@caas.cn

\begin{abstract}
NADC34-like PRRSV strains were first detected in China in 2017, with epidemic potential. In this study, the phylogenetic, epidemic, and recombinant properties of NADC34-like PRRSV in China were evaluated comprehensively. From 2020 to October 2021, 82 NADC34-like PRRSV isolates were obtained from 433 PRRSV-positive clinical samples. These strains accounted for $11.5 \%$ and $28.6 \%$ of positives in 2020 and 2021, respectively, and have spread to eight provinces. We selected 15 samples for whole-genome sequencing, revealing genome lengths of 15,009 to 15,113 nt. Phylogenetic analysis revealed that Chinese NADC34-like strains cluster with American sublineage 1.5 strains and do not form an independent branch. Recombination analysis revealed that six of fifteen complete genome sequences derived from recombination between NADC34-like and NADC30-like or HP-PRRSV; they all recombined with local strains in China, exhibiting a complex recombination pattern. Partial Nsp2 sequence alignment showed that nine of fifteen isolates have a continuous 100-aa deletion (similar to IA/2014/NADC34); other isolates have a 131-aa discontinuity deletion (similar to NADC30). Five of them also have additional amino acid deletions, all of which are reported for the first time here. In the last two years, NADC34-like PRRSV has become one of the main epidemic strains in some areas of China; it has changed significantly, its homology has decreased significantly, and it has undergone complex recombination with local Chinese strains. These results are of great significance for understanding the current epidemic situation of PRRSV in China.
\end{abstract}

\title{
Keywords:
}

NADC34-like PRRSV, novel characterization, recombination, endemic strain

\section{Introduction}

Porcine reproductive and respiratory syndrome (PRRS) is a major disease causing significant economic losses in the global swine industry. The causative agent, porcine reproductive and respiratory syndrome virus (PRRSV), is an enveloped, positive-sense, single-stranded RNA virus belonging to the family Arteriviridae of the order Nidovirales (Lunney et al., 2016). The PRRSV genome is approximately $15 \mathrm{~kb}$ in length, encoding at least 10 open reading frames (ORFs) (Firth et al., 2011). PRRSV can be divided into type 1 (PRRSV-1) and type 2 (PRRSV-2) based on genetic and antigenic differences (Murtaugh et al., 2010). Recently, PRRSV-1 was classified as Betaarterivirus suid 1, and PRRSV-2 was designatedBetaarterivirus suid 2 (ICTV2021). In China, PRRSV-2 strains have been circulating and predominate on farms since their initial emergence in 1996. Based on the global PRRSV classification system and ORF5 sequence, PRRSV-2 is divided into nine lineages (1-9) with several sublineages in each (Shi et al., 2010a; Shi et al., 2010b).

Before 2020, an overwhelming majority of PRRSV-2 strains in China could be divided into four lineages: sublineage 8.7 (JXA1-like and CH-1a-like), sublineage 5.1 (VR-2332-like), sublineage 3.5 (QYYZ-like) and sublineage 1.8 (NADC30-like) (Gao et al., 2017). However, in 2019, NADC34-like PPRSV was reported in China to be a potential pandemic strain (Xu et al., 2020). This kind of strain was initially reported in the United States in 2014, with dramatic abortion storms in sows and high mortality rates in piglets (van Geelen et al., 2018). This strain became endemic in Peru in 2015-2017 and had a severe impact on the local swine industry (Ramirez et al., 2019). The first NADC34-like PRRSV in China was reported in Liaoning Province in 2018 (Zhang et al., 2018); it carried a genetic marker of a 100-aa deletion corresponding to position 328427 of the Nsp2 gene of VR2332 (Bao and Li, 2021). Since then, several studies have demonstrated spread of 
NADC34-like PRRSV in at least four different regions of China (Heilongjiang, Liaoning, Henan and Fujian) (Liu et al., 2019; Xie et al., 2020a; Xu et al., 2020; Zhang et al., 2018). The pathogenicity of different NADC34-like strains varies greatly. The American strain IA/2014/NADC34 exhibits high pathogenicity (van Geelen et al., 2018); in contrast, the Chinese strains PRRSV-ZDXYL-China-2018-1 and HLJDZD321901 have moderate and low pathogenicity, respectively (Song et al., 2020; Xie et al., 2020b).

Our lab has been monitoring PRRSV for a long time, and the detection rate of NADC34-like strains increased significantly in 2020 and 2021. On farms with cases, severe miscarriage and other clinical symptoms typically occur. To further study the complete genome characteristics of NADC34-like PRRSV in China, we selected fifteen NADC34-like strains based on large homology differences and different branches of the phylogenetic tree based on whole-genome sequencing. The prevalence and molecular characteristics of NADC34 PRRSV in China were analyzed in detail.

\section{Materials and Methods}

From 2020 to October 2021, we collected 828 clinical samples (including serum, lymph node and lung samples) of suspected PRRSV infection from different pig farms in 14 provinces in China (Heilongjiang, Jilin, Liaoning, Shandong, Shanxi, Henan, Jiangxi, Anhui, Guangdong, Guangxi, Hebei, Hubei, Xinjiang and Jiangsu). Tissue sample disposal, RNA extraction, cDNA preparation, RT-PCR and genome sequencing were conducted as described previously (Leng et al., 2014; Zhang et al., 2015). The primers used to detect PRRSV and amplify entire gene sequences have been reported (Zhang et al., 2018).

The complete genome and different regions of the genome for each NADC34-like PRRSV isolate were compared with representative strains using ClustalW in Lasergene software (DNASTAR Inc., Madison, USA). To assess Nsp2 deletion patterns of the isolates, amino acid alignments were conducted between the isolates and reference strains using ClustalW in Lasergene software.

To understand the evolutionary characteristics of novel NADC34-like isolates, phylogenetic trees were constructed based on complete genomic and ORF5 nucleotide sequences, together with the representative strain PRRSV-2. Phylogenetic trees and molecular evolutionary analyses were carried out with MEGA 7.0 software using the neighbor-joining method and the Kimura 2-parameter substitution model and bootstrap tests of 1000 replicates (Kumar et al., 2016).

To test for recombination, the alignment was screened using RDP4 software (Murtaugh et al., 2010) as described in Zhang et al. (Zhang et al., 2019). Potential recombination events were tested by seven different algorithms (RDP, GeneConv, BootScan, MaxChi, Chimera, SiScan, and 3Seq) with Bonferroni correction. Only recombination events detectable using four or more of the seven methods implemented in RDP4 were taken as significant evidence of recombination. Recombination events were detected using Simplot v 3.5.1, and boot scanning analysis was performed with a 200-bp window, sliding along the genome alignment with a step size of $20 \mathrm{bp}$.

Results and Discussion

From 2020 to October 2021, 828 clinical samples were collected from 14 provinces of China; 433 (52.29\%) tested positive for PRRSV by RT-PCR. Through sequence alignment and phylogenetic analysis, 28 and 54 NADC34-like PRRSV samples were confirmed in 2020 and 2021, respectively (Fig. 1b), and 78 ORF5, 1 partial ORF7 and 3 partial Nsp2 gene sequences were successfully obtained (Table 1). To compare the epidemic trend of these strains, the number and proportion of NADC34 strains detected in China from 2017 to 2021 were plotted based on previous studies (Xu et al., 2020) (Fig. 1). As shown in Fig. 1a, the proportion of such strains was less than $3 \%$ in 2017-2019, skyrocketed to $11.5 \%$ in 2020, and reached a staggering $28.6 \%$ in 2021 (Fig. 1a). In 2021, NADC34-like PRRSV became one of the major circulating strains in parts of China, along with NADC30-like (35.4\%) and HP-PRRSV (31.2\%). All positive samples of the NADC34like strains were from Heilongjiang, Jilin, Liaoning, Hebei, Shandong, Henan and Jiangsu provinces, and this lineage was first detected in some of these provinces (Table 1). The above results demonstrate that NADC34-like PRRSV has become one of the main epidemic strains in some regions of China and has spread 
to more provinces. Hence, it is urgent to strengthen monitoring of these strains. To better understand the genetic relationship and evolution of NADC34-like PRRSV in China, we selected 15 positive samples for whole-genome sequencing. The genomes of these isolates were 15,009-15,113 nt in length, excluding 3' poly (A) tails.

To understand the evolutionary relationships of all new NADC34-like PRRSV strains with representative strains, phylogenetic trees were constructed using the neighbor-joining method. According to the phylogenetic tree based on GP5, all isolated strains can be classified as sublineage 1.5, and these strains can be divided into five groups (Fig. 2a). All strains classified as Group 5 recombined with sublineage 1.8 (NADC30-like) strains, divided into Group 5a and Group 5b based on different recombination locations. To analyze the genetic diversity of sublineage 1.5 PRRSV in as much detail as possible, we expanded a data set $(2,202)$ including all ORF5 sequences of sublineage 1.5 collected from a recent NCBI update. As shown in Fig. 2b, all five groups of NADC34-like strains in China cluster deep within sublineage 1.5 from the United States. They do not form a separate clade, suggesting that the NADC34-like strains in China may derive not only from the spread of earlier similar strains but also from continued importation of strains from the United States. Whole-genome sequencing data would unveil a broader perspective on PRRSV evolution (Paploski et al., 2021). When phylogenetic trees were constructed based on whole-genome gene sequences, the HLJTZJ2007-2106, JLTZJ2050-2107, HLJTZJ2090-2107 and HLJTZJ2165-2108 isolates were classified as sublineage 1.8 (NADC30-like); however, they belong to sublineage 1.5 based on ORF5 sequence (Fig. 2c). This is the first report of Chinese NADC34-like strains classifying into sublineage 1.8 based on genome-wide phylogenetic analysis. These results emphasize the disadvantages of relying only on ORF5 sequence typing and indicate the emergence of new recombinant strains.

Recombination is a pervasive phenomenon among PRRSV isolates and is an important strategy for generating viral genetic diversity (Chen et al., 2013). Chinese NADC34-like PRRSV reportedly shows a complex recombination pattern but as of 2019, it had not recombined with local strains in China (Xu et al., 2020). To monitor current recombination of this strain in China, RDP4 and SimPlot software were used to assess possible recombinant events. Recombination events were observed in six of fifteen whole-genome sequences (Table 2). Three recombinant isolates (HLJPY18-2009, HLJTZJ2007-2106 and HLJTZJ2165-2108) emerged from the recombination event between HP-PRRSV isolates, NADC30-like virus and NADC34-like virus; the three recombinant isolates (HLJTZJ1988-2106, JLTZJ2050-2107 and HLJTZJ2090-2107) also derived from NADC30-like PRRSV and NADC34-like PRRSV (Table 2, Fig. S1). These putative recombinant events were further supported by statistically incongruent phylogenetic trees (Fig. S1). According to a similarity plot, these strains have an extremely complex recombination pattern (Table 2, Fig. S1), with most of the breakpoints of recombination events in the six strains being located in Nsp1(4), Nsp2(4), Nsp3(1), Nsp4(1), Nsp6(1), Nsp7(1), Nsp9(2), Nsp10(2), Nsp12(2), ORF2(2), ORF4(1), ORF5(3), ORF6(2), and ORF7(1). Of note, recombination events between NADC34-like virus and local strains of other lineages in China virus have emerged since 2020. Chinese HP-PRRSV provided partial recombinant fragments for HLJPY18-2009 (JX878380.1 SDA3), HLJTZJ2007-2106 (EF635006.1 HuN4) and HLJTZJ2165-2108 (HuN4). At the same time, BLAST analysis indicated that HLJTZJ1988-2106, JLTZJ2050-2107 and HLJTZJ2090-2107 most likely derive from the Chinese strains GXNN202004 (MW561594.1), QHD2 (MH167387.1) and HLJWK108-1711 (MN046230.1) (data not shown). We found that the earliest recombination event with the local strain occurred in September 2020 (HLJPY18-2009), consistent with the surge in the number and proportion of NADC34-like PRRSV isolates in 2020 to 2021. Therefore, recombination with the local strain may be one of the reasons for the rapidly developing epidemic of this strain in China. Frequent recombination events among PRRSV strains can significantly alter their pathogenicity. In the last quarter of 2020, veterinarians from the United States reported the presence of a highly pathogenic RFLP 1-4-4 lineage 1C variant strain (Trevisan et al., 2021), and this strain may be a recombinant strain with the NADC34-like strain as the main parent. Changes in the pathogenicity of NADC34-like strains recombined with local Chinese strains should be further investigated.

To examine genomic variation in nonrecombinant NADC34-like PRRSV isolates (HLJWK318-2001, HNTZJ165-2001, SDHSW135-2009, HLJTZJ829-2010, HLJTZJ864-2010, HLJTZJ921-2011, HLJTZJ1289- 
2012, LNTZJ1341-2012, HLJPY32-2109), nucleotide and amino acid (aa) homologies of the whole genome, 5'UTR, 3'UTR, ORF1a, ORF1b and ORF2-7 genes in the nonrecombinant strains and in two representative strains (IA/2014/NADC34, LNWK130) were compared. Complete genome sequence alignments revealed that NADC34-like isolates share 96.0-96.9\% identity with IA/2014/NADC34 and 93.8-94.3\% identity with LNWK130 (Table 3). Moreover, the homology between the latest NADC34-like strains and the first Chinese NADC34-like strain LNWK130 was significantly lower than that of the American representative strain IA/2014/NADC34, suggesting that the current epidemic of these lineage strains in China was not caused by mutation of the LNWK130 strain but by multiple inputs and separate evolution. A full genome alignment of all nonrecombinant NADC34-like sequences revealed marked variation, with nucleotide identities between all genomes varying from $93.2 \%$ to $99.9 \%$ (Table 3). The most variable region was that coding for Nsp2 (nucleotide homology from $90.2 \%$ to $99.8 \%$; amino acid homology from $86.9 \%$ to 100\%), and the most conserved region was Nsp9 (nucleotide homology from 95.1\% to 99.8\%; amino acid homology from $97.8 \%$ to $100 \%$ ), which encodes RNA-dependent RNA polymerase. The Nsp2 gene exhibits the highest genetic diversity among PRRSV strains (Nelsen et al., 1999). In 2020-2021, only 86.9\% amino acid homology was found in the Nsp2 region of undetected recombination strains, significantly lower than the $92.7 \%$ of the earlier strains (Xu et al., 2020). Therefore, NADC34-like strains undergo rapid mutation in the Nsp2 region.

Nsp2 can tolerate amino acid deletions and foreign gene insertions (Fang et al., 2004) and is used as a molecular marker to distinguish different strains, such as the linked 30-amino-acid (aa) discontinuous deletion in Nsp2 of the highly pathogenic PRRSV strain in China (An et al., 2011), the linked 131-aa discontinuous deletion in Nsp2 of NADC30-like PRRSV (Brockmeier et al., 2012), and the 100-aa continuous deletion in Nsp2 of NADC34-like PRRSV (van Geelen et al., 2018). Partial Nsp2 sequence alignment showed that all isolates in Group A harbor a 100-aa continuous deletion at residues 328-427 of VR-2332 Nsp2, which is consistent with LNWK130 and IA/2014/NADC34 (Fig. 3). Interestingly, Nsp2 of HLJWK318-2001, HNTZJ165-2001 and HLJPY32-2109 carry additional continuous deletions in the regions of residues 151152, 484-487 and 501-508, respectively, displaying novel mutation patterns $(2+100$ aa, $100+4$ aa and $100+8$ aa). Although the isolates in Group B show a 131-aa discontinuous deletion at aa 323-432, 483 and 504-522, which is similar to NADC30-like PRRSV, HLJTZJ2007-2106 has a 1-aa additional deletion at aa 805 and HLJTZJ2165-2108 a 3-aa additional deletion at aa 503 and 523-524 (Fig. 3). Different from the 100-aa continuous deletion of all NADC34-like strains prior to 2019, some newly identified NADC34-like strains from China showed a deletion pattern similar to NADC30-like strains, which was consistent with the results of recombinant analysis. Therefore, NADC34-like strains in China showed more complex deletion patterns in Nsp2 region, which also made it more difficult to classify the strains using deletion patterns.

Lineage 1 PRRSV, including sublineages 1.1-1.9, has a long epidemic history. These strains initially appeared in Canada before spreading through the United States (Shi et al., 2010b) and became the most prevalent and diverse lineage in the latter (Paploski et al., 2021). Since the first reports of sublineage 1.8 (NADC30-like) strains in 2013, the virus has rapidly spread to many regions of China, and researchers and farmers in China are beginning to pay more attention to lineage1 strains. Subsequently, a sublineage 1.5 (NADC34-like) strain was discovered in China in 2017 and rapidly became prevalent after 2020. The emergence of both lineage 1 strains in China was caused by import from the United States. Furthermore, lineage1 PRRSV is not only endemic in the United States and China. For example, in South Korea, lineage 1 comprised the second-largest population (29.6\%) of PRRSV in 2019 (Kim et al., 2021). In Peru, 75\% of the strains detected during 2015-2017 were associated with PRRSV 1-7-4 (NADC34-like) strains (Ramirez et al., 2019), and the most reported strain in the Canadian provinces of Ontario and Quebec belongs to lineage 1 (Ramirez et al., 2019). According to our data, lineage 1 (sublineage 1.5 and sublineage 1.8) strains accounted for $64 \%$ of positive samples in 2021, much higher than other lineages. Lineage 1 PRRSV has seriously affected the swine industry in China. At present, there is no vaccine for lineage 1 strains on the market worldwide, and the existing vaccine only provides partial protection against lineage 1 PRRSV (Bai et al., 2016). Therefore, while increasing monitoring of lineage 1 strains, it is also necessary to develop safe and effective vaccine prevention and to control lineage 1 strains.

Conclusion 
Since 2021, NADC34-like PRRSV has become one of the main epidemic strains in some regions of China. Furthermore, based on phylogenetic and whole-genome homology analyses, Chinese NADC34-like PRRSVs may derive from several sources. NADC34-like PRRSV has recombined with local strains in China and shows complex recombination. Novel changes in NADC34-like PRRSV pose a great challenge for monitoring as well as the prevention and control of PRRSV. Therefore, we should pay more attention to continuously monitoring NADC34-like PRRSV and strengthening PRRSV prevention and control.

\section{Acknowledgments}

This study was supported by grants from the National Natural Science Foundation of China (Grant no. 32172890 and 32002315), the China Postdoctoral Fund (Grant no. 2020M680788), the Natural Science Foundation of Heilongjiang Province (Grant no. YQ2019C032 and YQ2019C030), and the State Key Laboratory of Veterinary Biotechnology Foundation (Grant no. SKLVBF202115).

\section{Conflicts Of Interest}

The authors declare no potential conflicts of interest with respect to the research, authorship and publication of this article.

\section{References}

An, T.Q., Tian, Z.J., Leng, C.L., Peng, J.M., Tong, G.Z., 2011. Highly pathogenic porcine reproductive and respiratory syndrome virus, Asia. Emerg Infect Dis 17, 1782-1784.

Bai, X., Wang, Y., Xu, X., Sun, Z., Xiao, Y., Ji, G., Li, Y., Tan, F., Li, X., Tian, K., 2016. Commercial vaccines provide limited protection to NADC30-like PRRSV infection. Vaccine 34, 5540-5545.

Bao, H., Li, X., 2021. Emergence and spread of NADC34-like PRRSV in China. Transbound Emerg Dis.

Brockmeier, S.L., Loving, C.L., Vorwald, A.C., Kehrli, M.E., Jr., Baker, R.B., Nicholson, T.L., Lager, K.M., Miller, L.C., Faaberg, K.S., 2012. Genomic sequence and virulence comparison of four Type 2 porcine reproductive and respiratory syndrome virus strains. Virus Res 169, 212-221.

Chen, N., Yu, X., Wang, L., Wu, J., Zhou, Z., Ni, J., Li, X., Zhai, X., Tian, K., 2013. Two natural recombinant highly pathogenic porcine reproductive and respiratory syndrome viruses with different pathogenicities. Virus Genes 46, 473-478.

Fang, Y., Kim, D.Y., Ropp, S., Steen, P., Christopher-Hennings, J., Nelson, E.A., Rowland, R.R., 2004. Heterogeneity in Nsp2 of European-like porcine reproductive and respiratory syndrome viruses isolated in the United States. Virus Res 100, 229-235.

Firth, A.E., Zevenhoven-Dobbe, J.C., Wills, N.M., Go, Y.Y., Balasuriya, U.B.R., Atkins, J.F., Snijder, E.J., Posthuma, C.C., 2011. Discovery of a small arterivirus gene that overlaps the GP5 coding sequence and is important for virus production. J Gen Virol 92, 1097-1106.

Gao, J.C., Xiong, J.Y., Ye, C., Chang, X.B., Guo, J.C., Jiang, C.G., Zhang, G.H., Tian, Z.J., Cai, X.H., Tong, G.Z., An, T.Q., 2017. Genotypic and geographical distribution of porcine reproductive and respiratory syndrome viruses in mainland China in 1996-2016. Vet Microbiol 208, 164-172.

Kim, S.C., Jeong, C.G., Park, G.S., Park, J.Y., Jeoung, H.Y., Shin, G.E., Ko, M.K., Kim, S.H., Lee, K.K., Kim, W.I., 2021. Temporal lineage dynamics of the ORF5 gene of porcine reproductive and respiratory syndrome virus in Korea in 2014-2019. Arch Virol 166, 2803-2815.

Kumar, S., Stecher, G., Tamura, K., 2016. MEGA7: Molecular Evolutionary Genetics Analysis Version 7.0 for Bigger Datasets. Mol Biol Evol 33, 1870-1874.

Leng, C.L., Tian, Z.J., Zhang, W.C., Zhang, H.L., Zhai, H.Y., An, T.Q., Peng, J.M., Ye, C., Sun, L., Wang, Q., Sun, Y., Li, L., Zhao, H.Y., Chang, D., Cai, X.H., Zhang, G.H., Tong, G.Z., 2014. Characterization of 
two newly emerged isolates of porcine reproductive and respiratory syndrome virus from Northeast China in 2013. Vet Microbiol 171, 41-52.

Liu, J., Wei, C., Lin, Z., Xia, W., Ma, Y., Dai, A., Yang, X., 2019. Full genome sequence analysis of a 1-7-4-like PRRSV strain in Fujian Province, China. PeerJ 7, e7859.

Lunney, J.K., Fang, Y., Ladinig, A., Chen, N., Li, Y., Rowland, B., Renukaradhya, G.J., 2016. Porcine Reproductive and Respiratory Syndrome Virus (PRRSV): Pathogenesis and Interaction with the Immune System. Annu Rev Anim Biosci 4, 129-154.

Murtaugh, M.P., Stadejek, T., Abrahante, J.E., Lam, T.T., Leung, F.C., 2010. The ever-expanding diversity of porcine reproductive and respiratory syndrome virus. Virus Res 154, 18-30.

Nelsen, C.J., Murtaugh, M.P., Faaberg, K.S., 1999. Porcine reproductive and respiratory syndrome virus comparison: divergent evolution on two continents. J Virol 73, 270-280.

Paploski, I.A.D., Pamornchainavakul, N., Makau, D.N., Rovira, A., Corzo, C.A., Schroeder, D.C., Cheeran, M.C., Doeschl-Wilson, A., Kao, R.R., Lycett, S., VanderWaal, K., 2021. Phylogenetic Structure and Sequential Dominance of Sub-Lineages of PRRSV Type-2 Lineage 1 in the United States. Vaccines (Basel) 9 .

Ramirez, M., Bauermann, F.V., Navarro, D., Rojas, M., Manchego, A., Nelson, E.A., Diel, D.G., Rivera, H., 2019. Detection of porcine reproductive and respiratory syndrome virus (PRRSV) 1-7-4-type strains in Peru. Transbound Emerg Dis 66, 1107-1113.

Shi, M., Lam, T.T., Hon, C.C., Hui, R.K., Faaberg, K.S., Wennblom, T., Murtaugh, M.P., Stadejek, T., Leung, F.C., 2010a. Molecular epidemiology of PRRSV: a phylogenetic perspective. Virus Res 154, 7-17.

Shi, M., Lam, T.T., Hon, C.C., Murtaugh, M.P., Davies, P.R., Hui, R.K., Li, J., Wong, L.T., Yip, C.W., Jiang, J.W., Leung, F.C., 2010b. Phylogeny-based evolutionary, demographical, and geographical dissection of North American type 2 porcine reproductive and respiratory syndrome viruses. J Virol 84, 8700-8711.

Song, S., Xu, H., Zhao, J., Leng, C., Xiang, L., Li, C., Fu, J., Tang, Y.D., Peng, J., Wang, Q., Zhao, H., An, T., Cai, X., Zhang, H., Tian, Z.J., 2020. Pathogenicity of NADC34-like PRRSV HLJDZD32-1901 isolated in China. Vet Microbiol 246, 108727.

van Geelen, A.G.M., Anderson, T.K., Lager, K.M., Das, P.B., Otis, N.J., Montiel, N.A., Miller, L.C., Kulshreshtha, V., Buckley, A.C., Brockmeier, S.L., Zhang, J., Gauger, P.C., Harmon, K.M., Faaberg, K.S., 2018. Porcine reproductive and respiratory disease virus: Evolution and recombination yields distinct ORF5 RFLP 1-7-4 viruses with individual pathogenicity. Virology 513, 168-179.

Xie, C., Ha, Z., Nan, F., Zhang, Y., Zhang, H., Li, J., Zhang, P., Han, J., Zhang, H., Zhuang, X., Zhang, J., Lu, H., Jin, N., 2020a. Characterization of porcine reproductive and respiratory syndrome virus (ORF5 RFLP 1-7-4 viruses) in northern China. Microb Pathog 140, 103941.

Xie, C.Z., Ha, Z., Zhang, H., Zhang, Y., Xie, Y.B., Zhang, H., Nan, F.L., Wang, Z., Zhang, P., Xu, W., Han, J.C., Wen, S.B., Lu, H.J., Jin, N.Y., 2020b. Pathogenicity of porcine reproductive and respiratory syndrome virus (ORF5 RFLP 1-7-4 viruses) in China. Transbound Emerg Dis.

Xu, H., Song, S., Zhao, J., Leng, C., Fu, J., Li, C., Tang, Y.D., Xiang, L., Peng, J., Wang, Q., Zhao, H., An, T., Cai, X., Zhang, H., Tian, Z.J., 2020. A potential endemic strain in China: NADC34-like porcine reproductive and respiratory syndrome virus. Transbound Emerg Dis 67, 1730-1738.

Zhang, H., Leng, C., Feng, L., Zhai, H., Chen, J., Liu, C., Bai, Y., Ye, C., Peng, J., An, T., Kan, Y., Cai, X., Tian, Z., Tong, G., 2015. A new subgenotype 2.1d isolates of classical swine fever virus in China, 2014. Infect Genet Evol 34, 94-105.

Zhang, H.L., Zhang, W.L., Xiang, L.R., Leng, C.L., Tian, Z.J., Tang, Y.D., Cai, X.H., 2018. Emergence of novel porcine reproductive and respiratory syndrome viruses (ORF5 RFLP 1-7-4 viruses) in China. Vet 
Microbiol 222, 105-108.

Zhang, W.L., Zhang, H.L., Xu, H., Tang, Y.D., Leng, C.L., Peng, J.M., Wang, Q., An, T.Q., Cai, X.H., Fan, J.H., Tian, Z.J., 2019. Two novel recombinant porcine reproductive and respiratory syndrome viruses belong to sublineage 3.5 originating from sublineage 3.2. Transbound Emerg Dis 66, 2592-2600.

\section{Hosted file}

Figure1.docx available at https://authorea.com/users/452704/articles/550680-novelcharacteristics-of-chinese-nadc34-like-prrrsv-during-2020-2021

\section{Hosted file}

Figure2.docx available at https://authorea.com/users/452704/articles/550680-novelcharacteristics-of-chinese-nadc34-like-prrrsv-during-2020-2021

\section{Hosted file}

Figure3.docx available at https://authorea.com/users/452704/articles/550680-novelcharacteristics-of-chinese-nadc34-like-prrrsv-during-2020-2021

\section{Hosted file}

Table1.docx available at https://authorea.com/users/452704/articles/550680-novelcharacteristics-of-chinese-nadc34-like-prrrsv-during-2020-2021

\section{Hosted file}

Table2.docx available at https://authorea.com/users/452704/articles/550680-novelcharacteristics-of-chinese-nadc34-like-prrssv-during-2020-2021

\section{Hosted file}

Table3.docx available at https://authorea.com/users/452704/articles/550680-novelcharacteristics-of-chinese-nadc34-like-prrrsv-during-2020-2021 